\title{
Tennis Home-Training during First Italy Lockdown COVID-19: A Pilot Study in Young Athletes
}

\section{Running Title: Effect of Training at Home during Lockdown COVID-19}

\author{
Michela Montorsi ${ }^{1}$, Alessandro Solmi ${ }^{1}$, Alessandra Vezzoli ${ }^{2}$, Renato De Donato ${ }^{3,4}$, Gennaro D'Angelo ${ }^{2}$, \\ Simona Mrakic-Sposta ${ }^{1,2, *}$ \\ ${ }^{1}$ Department of Human Sciences and Promotion of the Quality of Life, San Raffaele Roma Open University, Italy \\ ${ }^{2}$ Institute of Clinical Physiology, National Research Council (IFC- CNR), Italy \\ ${ }^{3}$ Department of Biomedical Sciences for Health, School of Exercise Sciences, University of Milan, Italy \\ ${ }^{4}$ Heracles Gymnasium SSD S.r.l. Fortitudo, Italy
}

Received September 10, 2021; Revised October 20, 2021; Accepted November 21, 2021

\section{Cite This Paper in the following Citation Styles}

(a): [1] Michela Montorsi, Alessandro Solmi, Alessandra Vezzoli, Renato De Donato, Gennaro D'Angelo, Simona Mrakic-Sposta , "Tennis Home-Training during First Italy Lockdown COVID-19: A Pilot Study in Young Athletes," International Journal of Human Movement and Sports Sciences, Vol. 9, No. 6, pp. 1403 - 1419, 2021. DOI: 10.13189/saj.2021.090637.

(b): Michela Montorsi, Alessandro Solmi, Alessandra Vezzoli, Renato De Donato, Gennaro D'Angelo, Simona Mrakic-Sposta (2021). Tennis Home-Training during First Italy Lockdown COVID-19: A Pilot Study in Young Athletes. International Journal of Human Movement and Sports Sciences, 9(6), 1403 - 1419. DOI: 10.13189/saj.2021.090637.

Copyright $\bigcirc 2021$ by authors, all rights reserved. Authors agree that this article remains permanently open access under the terms of the Creative Commons Attribution License 4.0 International License

\begin{abstract}
COVID-19 pandemic has imposed an Italy national lockdown between 9 March and 3 May 2020, limiting the ability of individuals to exercise/training outdoors. The aim of this study was based on the construction of remote-personalized-training protocols in tennis and examined the effects of a home-based intervention. Sixteen young athletes, divided into four groups in accord with the level of play and the pre-existing technical-tactical preparation, were trained by tennis-coach using simple equipment (i.e., balance board, medicine ball, steps). Physiological parameters ( $\left.\mathrm{HR}, \mathrm{SaO}_{2}\right)$, the ability to execute technical gestures in conditions of stress/fatigue, development of adaptation and motor reaction skills, as well as eye-manual coordination; execution of movements at high speeds and precision, prevention of muscle and joint injuries, were monitoring. A questionnaire able to evaluate the subjective perception was administered. Athletes have demonstrated, by means of this latter, the beneficial effects of home-training during the lockdown. Concluding, the remote home-training, offered to athletes one option to maintain "normal" training practices, in view of a return to the tennis court. Furthermore, at today, in post-pandemic environments and behaviors, the results
\end{abstract}

suggest that remote home-training intervention may help foster meaningful improvements in young athletes, strengthening intrinsic/subjective motivation.

Keywords Pandemic, Remote-Home-Training, Tennis, COVID-19, Lockdown

\section{Introduction}

In 2020, the World Health Organization (WHO) declared the Public Health Emergency of International Concern in January and Pandemic of coronavirus disease (COVID-19) in March. Since then, world people's lives changed radically. At the end of July 2021, the global situation counted 195,886,929 confirmed cases and $4,189,148$ deaths, while in Italy there were, at the same date, 4,330,739 confirmed cases and 128,010 deaths (World Health Organization, WHO) [1]. COVID-19 had impact of on the life aspects of all people in every part of the world [2,3,4,5]. From, March 9 to May 3, 2020, the government of Italy imposed a national lockdown, 
restricting the movement of the population, except for necessity, work, and health circumstances. The "lockdown" measures, despite being widely approved by public opinion, were also described as the largest suppression of constitutional rights in the history of the republic (Costituzione Italiana) [6].

The lockdown restrictions have negative socio-psychological impacts both on mental and physical health (i.e., isolation, depression, anxiety, insomnia) and, as reported by Hawkley and Capitanio [7], long-term isolation creates stress, negative emotion, impaired cognition. Furthermore, Rodríguez-Rey et al. [8] in their study have reported that adverse psychological effects have occurred on Spanish people, and young people and women suffered more than the others. Moreover, during the COVID-19 lockdown, in southern Italy, a cross-sectional study confirms a negative impact on young and adults [9]. It has also been reported that physical inactivity during the COVID-19 pandemic lockdown was deleterious for the cardiovascular system [10], has increased sedentary behaviors [11], with poorer dietary choices and increased alcohol consumption [12,13].

The pandemic of coronavirus disease (COVID-19) leads to all Worldwide movement restrictions that have caused significant disruption to athlete's training and consequently sporting competitions $[14,15,16]$.

Many athletes were not able to perform sport-specific training, due to movement restrictions [17], especially in children, adolescents, and young adults [18]. Many athletes have trained at home, often without specialist equipment or supervision [13,19].

In contrast, other athletes opportunistically used the 'lockdown' time during the pandemic in a positive way, to 'upskill' perhaps in preparation for their lives after sport [20,21]. Despite emerging evidence, demonstrating beneficial effects of home-training, the overall effectiveness of home-training for maintaining optimal levels of sport-specific conditioning remains unclear [22].

It is well known that sports practice has beneficial effects not only on the physical body but also on mental care [23, 24]. According to Garber et al. [25] and based on the recommendation of the American College of Sports Medicine, exercise improves physical and mental health and/or fitness in most persons without taking account of their training habits. It has also been reported that home-based tactics could include aerobic activities, balance and flexibility exercises, muscular strength and endurance training [26].

Recently, Loh et al. [27] have reported a positive effect of a home-based exercise program on social and emotional well-being among older patients with cancer and likewise, positive effects in people with chronic heart failure after individualized home-based physical program [28]. Moreover, it has been demonstrated that physical activity has beneficial effects in neurodegenerative diseases [29] and in older individuals to improve muscle mass and function $[30,31]$.

Tennis is one of the most popular sports in the world, with $1.12 \%$ of the world's population (about 75 million) participating in tennis. Tennis is played at every level and at any age from 4 to over 80 years old. It is therefore considered a valuable sport for maintaining healthy physical activity levels in middle aged and older adults [32].

Tennis is a highly specific sport that requires specific spaces [33] and beneficial effects on the musculoskeletal function of tennis players respected to non-players are reported. It is a static and dynamic sport and it has many health benefits: it increases brain power, improves handeye coordination, reduces stress, decreases cardiovascular disease, increases fitness levels, leads to stronger bones and muscles [34]. Tennis playing requires cognitive control, visuo-spatial orientation and specific tennis motor skills for efficient decisions [35]. Furthermore, tennis players with enhanced decision-making skills can use movement-pattern information to determine shot selection, reduce their response delay times and, hence, improve their stroke performance [36].

The idea of this study was based on the construction of personalized training protocols, elaborated and differentiated according to different parameters, such as the level of play of the participants, the availability of space in one's home, the availability of equipment and tools suitable for training suited to their characteristics, as well as the technical-metabolic needs required for maintaining good physical-technical - tactics, with a view to a future return to activities on the court. Each student was allowed to train directly with tennis coaches, remotely, each in their own homes, adapting protocols to the technical material and environments available to the various athletes. Then, the aim of this study was to find a solution to fight this constant and forced motor decline in daily life, during the COVID-19 lockdown in 2020.

\section{Materials and Methods}

\section{Subjects}

This study was carried out during the Italian lockdown and it involved 16 volunteers' young athletes, mean age 18.5 \pm 2.1 years (14 males: $18.4 \pm 2.2$ years 2 females: 19 years) of a tennis club (Modena, Italy). Anthropometric measures: height, weight, and BMI were assessed and are reported in Table 1.

The athletes have been divided in four groups, according to the level of play and the pre-existing technical-tactical preparation. Different activities were carried out to satisfy the physical-technical-tactical demands of all those involved, adapting the workouts to their needs and characteristic (Table 2). 
Table 1. Anthropometric parameters: height $(\mathrm{cm})$, weight $(\mathrm{kg})$ and Body Mass Index (BMI, kg. $\left.\mathrm{m}^{-2}\right)$ collected from the young athletes at pre (T1), during remote-personalized-home-training protocols (30 March - T2, and 20 April -T3) and at the end of the training (T4)

\begin{tabular}{|c|c|c|c|c|c|c|c|c|c|c|c|c|c|c|}
\hline \multirow{2}{*}{ Group } & \multirow{2}{*}{ Athletes } & \multirow{2}{*}{ Age } & \multicolumn{4}{|c|}{ Height (cm) } & \multicolumn{4}{|c|}{ Weight (kg) } & \multicolumn{4}{|c|}{ BMI $\left(\mathrm{kg} . \mathrm{m}^{-2}\right)$} \\
\hline & & & $T 1$ & $T 2$ & $T 3$ & $T 4$ & $T 1$ & $T 2$ & $T 3$ & $T 4$ & $T 1$ & $T 2$ & $T 3$ & $T 4$ \\
\hline \multirow{4}{*}{ A } & 1 & 16 & 167 & 167,2 & 167,5 & 167,9 & 60 & 61,2 & 61,5 & 62 & 21,5 & 21,6 & 21,9 & 21,9 \\
\hline & 2 & 19 & 178 & 178 & 178 & 178 & 76 & 76,5 & 75,9 & 75,7 & 24 & 24 & 23,7 & 23,7 \\
\hline & 3 & 17 & 171 & 171,2 & 171,2 & 171,2 & 65 & 66 & 66,6 & 66,6 & 22,6 & 22,6 & 22,6 & 22,6 \\
\hline & 4 & 16 & 179 & 179,4 & 180,1 & 180,1 & 73 & 73,5 & 74,4 & 74,5 & 22,8 & 22,8 & 22,9 & 22,9 \\
\hline \multirow{4}{*}{$\mathrm{B}$} & 5 & 20 & 175 & 175 & 175,1 & 175,1 & 70 & 68 & 67,9 & 67 & 22,9 & 22,2 & 21,9 & 21,9 \\
\hline & 6 & 19 & 165 & 165 & 165,1 & 165,1 & 52 & 52,2 & 52,5 & 52,4 & 19,1 & 19,1 & 19,1 & 19,1 \\
\hline & 7 & 20 & 182 & 182 & 182 & 182 & 77 & 77,5 & 78 & 77,4 & 23 & 23 & 23,3 & 23,2 \\
\hline & 8 & 18 & 174 & 174 & 174,2 & 174,2 & 73,4 & 74 & 73,2 & 72 & 24 & 24,4 & 24,4 & 24,8 \\
\hline \multirow{4}{*}{$\mathrm{C}$} & 9 & 18 & 175 & 175 & 176 & 176,3 & 71 & 71,3 & 73 & 73,5 & 23,2 & 23,2 & 23,6 & 23,6 \\
\hline & 10 & 20 & 182 & 182 & 182 & 182 & 75,5 & 76,9 & 77 & 77 & 22,6 & 22,9 & 22,9 & 22,9 \\
\hline & 11 & 16 & 169 & 169,5 & 171 & 171 & 60 & 61 & 61,7 & 62 & 21 & 21,4 & 20,9 & 21,2 \\
\hline & 12 & 24 & 177 & 177 & 177 & 177 & 73 & 74 & 75,1 & 75 & 23,3 & 23,3 & 23,9 & 23,9 \\
\hline \multirow{4}{*}{$\mathrm{D}$} & 13 & 18 & 188 & 188 & 188,5 & 188,5 & 87 & 87,5 & 88 & 87,4 & 24,6 & 24,6 & 24,9 & 24,6 \\
\hline & 14 & 19 & 170 & 170 & 170 & 170 & 66 & 65 & 65 & 64,5 & 22,8 & 22,3 & 22,5 & 22,1 \\
\hline & 15 & 16 & 173 & 173,5 & 174 & 174 & 71 & 71 & 70,5 & 69,8 & 23,7 & 23,7 & 23,1 & 22,8 \\
\hline & 16 & 20 & 177 & 177 & 177 & 177 & 75 & 74,5 & 74,5 & 73,7 & 23,9 & 23,6 & 23,6 & 23,3 \\
\hline
\end{tabular}

Table 2. Four groups of athletes (beginner, intermediate, and two advanced) in according to the level of play and the pre-existing technical-tactical preparation were done. Physiological parameters: HR: heart rate at rest; Theoretic HRmax (calculated by Tanaka formula: 208-0.7*age); HRmax (maximum 300mt speed test); and Reserve HR (calculated by: HRmax-HR at rest)

\begin{tabular}{|c|c|c|c|c|c|}
\hline Group & Athletes & $\begin{array}{l}\text { TheoreticHRmax } \\
\text { (Tanaka formula) }\end{array}$ & HRrest & $\begin{array}{c}\text { HRmax } \\
\text { (maximum } 300 \text { speed test) }\end{array}$ & $\begin{array}{c}\text { Reserve HR } \\
\text { (HRmax -HR rest) }\end{array}$ \\
\hline \multirow{4}{*}{ A } & 1 & 196,8 & 84 & 192 & 108 \\
\hline & 2 & 194,7 & 94 & 193 & 99 \\
\hline & 3 & 196,1 & 82 & 195 & 113 \\
\hline & 4 & 196,8 & 79 & 194 & 115 \\
\hline \multirow{4}{*}{ B } & 5 & 194 & 75 & 192 & 117 \\
\hline & 6 & 194,7 & 80 & 194 & 114 \\
\hline & 7 & 194 & 71 & 190 & 119 \\
\hline & 8 & 195,4 & 79 & 192 & 113 \\
\hline \multirow{4}{*}{$\mathrm{C}$} & 9 & 195,4 & 66 & 196 & 130 \\
\hline & 10 & 194 & 69 & 192 & 123 \\
\hline & 11 & 196,8 & 78 & 195 & 117 \\
\hline & 12 & 191,2 & 73 & 190 & 117 \\
\hline \multirow{4}{*}{$\mathrm{D}$} & 13 & 194,7 & 76 & 192 & 116 \\
\hline & 14 & 196,8 & 74 & 195 & 121 \\
\hline & 15 & 194 & 68 & 194 & 126 \\
\hline & 16 & 195,4 & 71 & 194 & 123 \\
\hline
\end{tabular}


Table 3. Training program of the beginner group (A)

\begin{tabular}{|c|c|c|c|c|c|}
\hline \multicolumn{6}{|c|}{ Group A - Beginner } \\
\hline WEEK & AIMS & EXECUTIVE METHODS & EXERCISES & $\begin{array}{l}\text { DURATION AND } \\
\text { REPETITIONS }\end{array}$ & RECOVERY \\
\hline $\begin{array}{c}1 \\
2^{\text {nd }}-8^{\text {th }} \text { March } \\
2020\end{array}$ & $\begin{array}{l}\text { ankle, elbow, wrist extensor muscles strengthening; } \\
\text { aerobic capacity; speed; motor reaction capacity; } \\
\text { mobility of shoulder, low arts; lumbar spine }\end{array}$ & $\begin{array}{l}\text { dumbbells; jump rope; tennis } \\
\text { balls; stretching }\end{array}$ & $\begin{array}{l}\text { extensions with dumbbells; } \\
\text { isometry; shuttle circuits; free body; } \\
\text { stretching exercises }\end{array}$ & $\begin{array}{c}2-3 \text { sets per exercise } \\
10 \text { repetitions per exercise } \\
15 \text { minutes total of stretching }\end{array}$ & $45 \mathrm{sec}-1 \mathrm{~min}$ \\
\hline $\begin{array}{c}2 \\
9^{\text {th }}-15^{\text {th }} \text { March } \\
2020\end{array}$ & $\begin{array}{l}\text { body stretching; extensor/flexor lower body muscles } \\
\text { strengthening; shoulder strengthening; tennis } \\
\text { technique practice; core work }\end{array}$ & $\begin{array}{l}\text { jump rope; tennis racquets; } \\
\text { free body work }\end{array}$ & $\begin{array}{l}\text { jumps with rope; stretching; } \\
\text { forehand/backhand }\end{array}$ & 2 round $45 \mathrm{sec}$ per exercise & $45 \mathrm{sec}-1 \mathrm{~min}$ \\
\hline $\begin{array}{c}3 \\
16^{\text {th }}-22^{\text {nd }} \text { March } \\
2020\end{array}$ & $\begin{array}{l}\text { lumbar spine \& lower arts stretching; circuits training } \\
\text { (cardio, tennis techniques circuit exercises; lower arts } \\
\text { strengthening; aerobic capacity) }\end{array}$ & $\begin{array}{l}\text { jump rope; tennis racquets; } \\
\text { medicine ball }\end{array}$ & $\begin{array}{l}\text { jumps with rope; stretching; } \\
\text { forehand/backhand }\end{array}$ & 2 round $45 \mathrm{sec}$ per exercise & $45 \mathrm{sec}-1 \mathrm{~min}$ \\
\hline $\begin{array}{c}4 \\
23^{\text {rd }}-29^{\text {th }} \text { March } \\
2020\end{array}$ & $\begin{array}{l}\text { cardio; abs workout; tennis techniques circuit } \\
\text { exercises; flexibility and mobility }\end{array}$ & $\begin{array}{l}\text { jump rope; tennis racquets; } \\
\text { tennis balls }\end{array}$ & $\begin{array}{l}\text { jumps with rope; stretching; } \\
\text { forehand/backhand/abs }\end{array}$ & 2 round $45 \mathrm{sec}$ per exercise & $45 \mathrm{sec}-1 \mathrm{~min}$ \\
\hline $\begin{array}{c}5 \\
30^{\text {th }} \text { March }-5^{\text {th }} \\
\text { April } 2020\end{array}$ & $\begin{array}{l}\text { cardio; tennis techniques circuit exercises; lower } \\
\text { body strengthening }\end{array}$ & $\begin{array}{l}\text { medicine ball; tennis } \\
\text { racquets; jump rope }\end{array}$ & $\begin{array}{l}\text { jumps; tennis serve \& slice; squats; } \\
\text { abs; push-ups }\end{array}$ & 2 round $45 \mathrm{sec}$ per exercise & $45 \mathrm{sec}$ \\
\hline $\begin{array}{c}6 \\
6^{\text {th }}-12^{\text {th }} \text { April } \\
2020 \\
\end{array}$ & $\begin{array}{l}\text { stretching; tennis serves; balance work; abs; tennis } \\
\text { techniques circuit exercises; lower body workout }\end{array}$ & $\begin{array}{l}\text { medicine ball; tennis } \\
\text { racquets; balance board }\end{array}$ & $\begin{array}{l}\text { push-ups; balance board isometry; } \\
\text { tennis serve; abs }\end{array}$ & 2 round $45 \mathrm{sec}$ per exercise & $45 \mathrm{sec}$ \\
\hline $\begin{array}{c}7 \\
13^{\text {th }}-19^{\text {th }} \text { April } \\
2020 \\
\end{array}$ & $\begin{array}{l}\text { lower body strengthening; abs workout; game tactical } \\
\text { tennis sequence; }\end{array}$ & $\begin{array}{l}\text { medicine ball; jump rope; } \\
\text { tennis racquets; }\end{array}$ & $\begin{array}{l}\text { crunch; squats; jumps; } \\
\text { forehand/backhand }\end{array}$ & 2 round $45 \mathrm{sec}$ per exercise & $45 \mathrm{sec}$ \\
\hline $\begin{array}{c}8 \\
20^{\text {th }}-26^{\text {th }} \text { April } \\
2020\end{array}$ & $\begin{array}{l}\text { cardio; shoulder work; abs workout; tennis serve \& } \\
\text { volley technique }\end{array}$ & $\begin{array}{l}\text { medicine ball; tennis } \\
\text { racquets; dumbbells; jump } \\
\text { rope }\end{array}$ & $\begin{array}{l}\text { jumps; side raises; abs crunches; } \\
\text { frontal squat; serve }\end{array}$ & 2 round $45 \mathrm{sec}$ per exercise & $30 \mathrm{sec}$ \\
\hline $\begin{array}{c}9 \\
27^{\text {th }} \text { April }-3^{\text {rd }} \\
\text { May } 2020\end{array}$ & balance work; cardio, lower body; mobility & jump rope; balance board & $\begin{array}{l}\text { balance board isometry; jumps; } \\
\text { crunches; squats }\end{array}$ & 2 round $45 \mathrm{sec}$ per exercise & $30 \mathrm{sec}$ \\
\hline
\end{tabular}


Table 4. Training program of the intermediate group (B)

\begin{tabular}{|c|c|c|c|c|c|}
\hline \multicolumn{6}{|c|}{ Group B - Intermediate } \\
\hline WEEK & AIMS & EXECUTIVE METHODS & EXERCISES & $\begin{array}{c}\text { DURATION AND } \\
\text { REPETITIONS }\end{array}$ & RECOVERY \\
\hline $\begin{array}{c}1 \\
\text { 2nd }-8 \text { th March } 2020\end{array}$ & $\begin{array}{l}\text { ankle, chest, triceps, low arts strengthening; aerobic } \\
\text { capacity; motor reaction capacity; coordination; } \\
\text { mobility }\end{array}$ & $\begin{array}{l}\text { dumbbells; medicine ball; jump } \\
\text { rope; tennis balls }\end{array}$ & $\begin{array}{l}\text { extensions with dumbbells; } \\
\text { squats; stretching; jumps }\end{array}$ & 3 round $15 \mathrm{sec}$ per exercise & $45 \mathrm{sec}-1 \mathrm{~min}$ \\
\hline $\begin{array}{c}2 \\
9 \text { th }-15 \text { th March } 2020\end{array}$ & cardio; stretching; forehand/backhand/serve; chest & $\begin{array}{l}\text { tennis racquets; technique } \\
\text { circuit; rope jump }\end{array}$ & $\begin{array}{l}\text { push ups; jumps; tennis circuit } \\
\text { sequences exercises }\end{array}$ & 3round $15 \mathrm{sec}$ per exercise & $45 \mathrm{sec}-1 \mathrm{~min}$ \\
\hline $\begin{array}{c}3 \\
16 \text { th }-22 \text { nd March } 2020 \\
\end{array}$ & $\begin{array}{l}\text { lower body strengthening; stretching; shoulder; } \\
\text { serve \& volley; abs workout }\end{array}$ & tennis racquets; dumbbells; & $\begin{array}{l}\text { push ups; squats; plank; tennis } \\
\text { circuit techniques }\end{array}$ & 3 round $15 \mathrm{sec}$ per exercise & $45 \mathrm{sec}$ \\
\hline $\begin{array}{c}4 \\
\text { 23rd }-29 \text { th March } 2020 \\
\end{array}$ & $\begin{array}{l}\text { shoulder mobility; cardio; abs workout; } \\
\text { forehand/backhand }\end{array}$ & medicine ball; rope jump; & $\begin{array}{l}\text { skip; jumps; mountain climber; } \\
\text { throws with ball }\end{array}$ & 3 round $15 \mathrm{sec}$ per exercise & $45 \mathrm{sec}$ \\
\hline $\begin{array}{c}5 \\
\text { 30th March }-5 \text { th April } \\
2020 \\
\end{array}$ & tennis technique; abs workout; cardio; chest & medicine ball; rope jump; & $\begin{array}{l}\text { full body crunch; jumps; ball } \\
\text { frontal throws; plank }\end{array}$ & 3 round $15 \mathrm{sec}$ per exercise & $45 \mathrm{sec}$ \\
\hline $\begin{array}{c}6 \\
6 \text { th }-12 \text { th April } 2020\end{array}$ & $\begin{array}{l}\text { cardio; biceps strengthening; serve } \& \text { smash } \\
\text { technique }\end{array}$ & $\begin{array}{l}\text { elastic bands; jump rope; } \\
\text { medicine ball; tennis racquet }\end{array}$ & $\begin{array}{l}\text { smash/serve; pike walk out; } \\
\text { jumps; squats; curls }\end{array}$ & 3 round $15 \mathrm{sec}$ per exercise & $30 \mathrm{sec}$ \\
\hline $\begin{array}{c}7 \\
\text { 13th }-19 \text { th April } 2020 \\
\end{array}$ & $\begin{array}{l}\text { explosive force; serve \& volley; balance; mobility; } \\
\text { lower body strengthening }\end{array}$ & $\begin{array}{l}\text { medicine ball; tennis racquets; } \\
\text { jump rope }\end{array}$ & $\begin{array}{l}\text { burpees; jumps; serve \& volley; } \\
\text { balance isometry }\end{array}$ & 3 round $15 \mathrm{sec}$ per exercise & $30 \mathrm{sec}$ \\
\hline $\begin{array}{c}8 \\
\text { 20th }-26 \text { th April } 2020\end{array}$ & $\begin{array}{l}\text { serve; forehand/backhand slice; cardio; aerobic } \\
\text { power; abs workout }\end{array}$ & $\begin{array}{l}\text { medicine ball; rope jump; } \\
\text { tennis racquets }\end{array}$ & $\begin{array}{l}\text { split step technique jumps; ball } \\
\text { frontal throws; crunch }\end{array}$ & 3round $15 \mathrm{sec}$ per exercise & $30 \mathrm{sec}$ \\
\hline $\begin{array}{c}9 \\
\text { 27th April - 3rd May } \\
2020\end{array}$ & volley; serve; abs; cardio; stretching & $\begin{array}{l}\text { medicine ball; tennis racquets; } \\
\text { jump rope }\end{array}$ & plank knee up; jumps; throws & 3 round $15 \mathrm{sec}$ per exercise & $30 \mathrm{sec}$ \\
\hline
\end{tabular}


Table 5. Training program of the advanced groups $(C$ and $D)$

\begin{tabular}{|c|c|c|c|c|c|}
\hline \multicolumn{6}{|c|}{ Groups $C$ and $D$ - Advanced } \\
\hline WEEK & AIMS & EXECUTIVE METHODS & EXERCISES & $\begin{array}{l}\text { DURATION AND } \\
\text { REPETITIONS }\end{array}$ & RECOVERY \\
\hline $\begin{array}{c}1 \\
2^{\text {nd }}-8^{\text {th }} \text { March } \\
2020\end{array}$ & $\begin{array}{l}\text { shoulder muscles strengthening; explosive force; core } \\
\text { abs; balance \& mobility; motor reaction capacity }\end{array}$ & $\begin{array}{l}\text { dumbbells; medicine ball; } \\
\text { tennis balls; balance board }\end{array}$ & $\begin{array}{l}\text { shoulder press; squats; ball throws; } \\
\text { balance board; stretching }\end{array}$ & $\begin{array}{c}3 \text { round } 45 \mathrm{sec} \text { per } \\
\text { exercise }\end{array}$ & $45 \mathrm{sec}$ \\
\hline $\begin{array}{c}2 \\
9^{\text {th }}-15^{\text {th }} \text { March } \\
2020\end{array}$ & $\begin{array}{l}\text { cardio; stretching; low body strengthening; lumbar } \\
\text { spine \& gluteus strengthening; }\end{array}$ & $\begin{array}{l}\text { elastic bands; jump rope; } \\
\text { medicine ball }\end{array}$ & $\begin{array}{l}\text { push ups; throws; squats; } \\
\text { forehand/backhand with no racquets }\end{array}$ & $\begin{array}{l}3 \text { round } 45 \mathrm{sec} \text { per } \\
\text { exercise }\end{array}$ & $45 \mathrm{sec}$ \\
\hline $\begin{array}{l}3 \\
16^{\text {th }}-22^{\text {nd }} \text { March } \\
2020 \\
\end{array}$ & $\begin{array}{l}\text { forehand; backhand volley; abs workout; core \& lower } \\
\text { body strengthening }\end{array}$ & tennis racquets; jump rope & jumps; wall squat; shoulder tap; plank & $\begin{array}{l}3 \text { round } 45 \mathrm{sec} \text { per } \\
\text { exercise }\end{array}$ & $30 \mathrm{sec}$ \\
\hline $\begin{array}{c}4 \\
23^{\text {rd }}-29^{\text {th }} \text { March } \\
2020\end{array}$ & $\begin{array}{l}\text { types of forehand; abs workout; balance; calf } \\
\text { strengthening }\end{array}$ & tennis racquets; balance board & $\begin{array}{l}\text { frontal squat; plank jacks; isometry; } \\
\text { calf roll backs }\end{array}$ & $\begin{array}{l}3 \text { round } 45 \mathrm{sec} \text { per } \\
\text { exercise }\end{array}$ & $30 \mathrm{sec}$ \\
\hline $\begin{array}{c}5 \\
30^{\text {th }} \text { March }-5^{\text {th }} \\
\text { April } 2020\end{array}$ & cardio; backhand types; explosive force; & $\begin{array}{l}\text { tennis racquets; medicine ball; } \\
\text { jump rope }\end{array}$ & $\begin{array}{l}\text { jumps; explosive skip; squats; ball } \\
\text { throws; burpees }\end{array}$ & $\begin{array}{l}3 \text { round } 45 \mathrm{sec} \text { per } \\
\text { exercise }\end{array}$ & 30sec \\
\hline $\begin{array}{c}6 \\
6^{\text {th }}-12^{\text {th }} \text { April } \\
2020\end{array}$ & $\begin{array}{l}\text { serve \& slices; cardio; shoulder \& up body } \\
\text { strengthening; stretching }\end{array}$ & $\begin{array}{l}\text { elastic bands; medicine ball; } \\
\text { jump rope; tennis racquets }\end{array}$ & $\begin{array}{l}\text { jumps; high throws; plank to push up; } \\
\text { side raises }\end{array}$ & $\begin{array}{l}3 \text { round } 45 \mathrm{sec} \text { per } \\
\text { exercise }\end{array}$ & $30 \mathrm{sec}$ \\
\hline $\begin{array}{c}7 \\
13^{\text {th }}-19^{\text {th }} \text { April } \\
2020\end{array}$ & $\begin{array}{l}\text { explosive force; top-spin forehand/backhand/ volley; } \\
\text { abs workout; cardio }\end{array}$ & $\begin{array}{l}\text { jump rope; tennis racquets; } \\
\text { medicine ball; dumbbells }\end{array}$ & $\begin{array}{l}\text { wall static squat; jumps; throws; } \\
\text { lateral run with dumbbells }\end{array}$ & $\begin{array}{l}3 \text { round } 45 \mathrm{sec} \text { per } \\
\text { exercise }\end{array}$ & 30sec \\
\hline $\begin{array}{c}8 \\
20^{\text {th }}-26^{\text {th }} \text { April } \\
2020 \\
\end{array}$ & low body explosive strengthening; cardio; abs; balance & $\begin{array}{l}\text { tennis racquets; jump rope; } \\
\text { medicine ball }\end{array}$ & $\begin{array}{l}\text { skip; jumps; ball throws; planks; free } \\
\text { dribbles with tennis ball }\end{array}$ & $\begin{array}{l}3 \text { round } 45 \mathrm{sec} \text { per } \\
\text { exercise }\end{array}$ & $30 \mathrm{sec}$ \\
\hline $\begin{array}{c}9 \\
27^{\text {th }} \text { April }-3^{\text {rd }} \\
\text { May } 2020\end{array}$ & $\begin{array}{l}\text { low body strengthening; abs workout; cardio; motor } \\
\text { reaction capacity improvement }\end{array}$ & $\begin{array}{l}\text { tennis racquets \& balls; jump } \\
\text { rope; medicine ball }\end{array}$ & $\begin{array}{l}\text { jumps; frontal squat; throws; burpees; } \\
\text { plank; crunches }\end{array}$ & $\begin{array}{l}3 \text { round } 45 \mathrm{sec} \text { per } \\
\text { exercise }\end{array}$ & $30 \mathrm{sec}$ \\
\hline
\end{tabular}


Briefly summarizing the temporal structure of the study, it is possible to say that the 4 groups, of 4 subjects each, had the opportunity to train, 2 times a week, for a total period of 9 weeks which covered approximately the entire duration of the national lockdown, from 2 March to 4 May 2020. The surveys were carried out at: 2 March (T1), 30 March (T2), 20 April (T3), 2 May (T4) 2020.

Tables 3, 4 and 5 describe the training program of the beginner group (A), the intermediate group (B) and the advanced groups (C and D). Each table describes the aims of the training for each week of the lockdown and the executive methods, the exercises, the duration and repetitions and the recovery time.

The training was obviously provided by a connection with "Video-Call" mode through the Zoom or Microsoft Teams platforms, with each group connected independently with the undersigned on their own dedicated weekly day.

The equipment included: Dunlop Fort All Court $3 \& 4$ tennis balls ("regular" type, used in tennis teaching from 14 years upwards); Balance Board (proprioception and balance training); Fitness elastic bands; Skipping Node; Medicine ball -3 or $5 \mathrm{~kg}$; Mat; Chairs; Weights (dumbbells) varying from 1 to $5 \mathrm{~kg}$; Steps or, as alternative, stairs.

The equipment used to monitor some parameters related to training even during the performance of the activity were:

- Apple Watch series 4 (HR, FCmax, $\mathrm{VO}_{2}$ parameter monitoring)

- Electronic device (PC, Tablet, Smartphone) to periodically connect remotely to the training session (videocalls made through the Zoom platform or Microsoft Teams)

- Preset digital stop watch on Apple Watch series 4

- Finger Clips $\mathrm{O}_{2}$ saturation $\left(\mathrm{SaO}_{2}\right)$

Remote training protocols have been customized according to the level of play and the technical-physical-tactical characteristics of each athlete. There are three fundamental points to which a tennis-coach must pay particular attention:

I. Ability to execute technical gestures in conditions of stress and fatigue, as well as a strong development of adaptation and motor reaction skills, as well as eye-manual coordination;

II. Execution of movements at high speeds that require maximum precision at the same time;

III. Importance in the prevention of muscle and joint injuries.

The training evaluated parameters and the specific methodology are showed in Table 6 and 7.

Table 6. Type of exercise and training evaluated parameters

\begin{tabular}{|c|c|c|c|}
\hline TRAINING GOALS & GENERAL TRAINING & SPECIAL TRAINING & SPECIFIC TRAINING \\
\hline \multicolumn{4}{|c|}{ Tactical and Technical skills } \\
\hline - $\quad$ Technical-tactical memory & & & $\begin{array}{l}\text { Exercise with racket } \\
\text { Work on the execution of } \\
\text { technical tennis shots } \\
\text { Short game tactical sequences }\end{array}$ \\
\hline \multicolumn{4}{|c|}{ Conditional skills } \\
\hline $\begin{array}{ll}- & \text { Muscle strength and power } \\
\text { - } & \text { Sports speed and agility } \\
\text { - } & \text { Aerobic and muscle endurance }\end{array}$ & $\begin{array}{l}\text { Weight resistance training } \\
\text { Body-weight resistance } \\
\text { training } \\
\text { CORE training } \\
\text { Functional step training }\end{array}$ & $\begin{array}{l}\text { Interval training } \\
\text { High-intensity resistance } \\
\text { interval training }\end{array}$ & $\begin{array}{l}\text { Short game sequences (game } \\
\text { tactics with quick movements) }\end{array}$ \\
\hline \multicolumn{4}{|c|}{ Coordination skills } \\
\hline $\begin{array}{ll}\cdot & \text { Balance ability } \\
- & \text { Muscle reaction ability } \\
\text { - } & \text { Eye-manual and hand-foot } \\
& \text { coordination } \\
\text { - } & \text { Motor combination } \\
\text { - } & \text { Rhythm capacities } \\
\end{array}$ & $\begin{array}{l}\text { Balance board training } \\
\text { Body balance training } \\
\text { Jump rope }\end{array}$ & $\begin{array}{l}\text { Body balance training with } \\
\text { tennis ball } \\
\text { Complex exercise with } \\
\text { medicine ball }\end{array}$ & $\begin{array}{l}\text { Practice with tennis ball (i.e. } \\
\text { throwing against the wall) } \\
\text { Tennis technique and tactics }\end{array}$ \\
\hline
\end{tabular}


Table 7. Specific methodology adopted for the athletes of the different groups.

\begin{tabular}{|c|c|c|c|c|c|c|c|c|c|c|}
\hline \multicolumn{6}{|c|}{ DAILY WARM UP FOR ALL THE GROUPS } & \multicolumn{5}{|c|}{ DAILY WASH OUT FOR ALL THE GROUPS } \\
\hline \multicolumn{2}{|c|}{ Type of Exercise } & \multicolumn{4}{|c|}{ Time } & \multicolumn{2}{|c|}{ Type of Exercise } & \multicolumn{3}{|c|}{ Time } \\
\hline \multicolumn{2}{|c|}{ Shoulder mobility } & \multicolumn{4}{|c|}{10 minutes } & \multicolumn{2}{|c|}{ Juggling with tennis ball } & \multicolumn{3}{|c|}{10 minutes } \\
\hline \multicolumn{2}{|c|}{ Balance exercise } & \multicolumn{4}{|c|}{10 minutes } & \multicolumn{2}{|c|}{ Static stretching } & \multicolumn{3}{|c|}{10 minutes } \\
\hline \multicolumn{2}{|c|}{ Dynamic flexibility } & \multicolumn{4}{|c|}{10 minutes } & \multicolumn{2}{|c|}{ Cognitive task } & \multicolumn{3}{|c|}{5 minutes } \\
\hline \multicolumn{11}{|c|}{ Group A - Beginner } \\
\hline \multicolumn{11}{|c|}{ PREPARATORY PHASE } \\
\hline \multicolumn{6}{|c|}{ TRAINING 1} & \multicolumn{5}{|c|}{ TRAINING 2} \\
\hline \multirow{6}{*}{$1-3$ weeks } & Exercises & Reps & Set & $\operatorname{Rec}$ & Load & Exercises & Reps & Set & $\operatorname{Rec}$ & Load \\
\hline & Air Squat & 15 & 4 & $1^{\prime}$ & BW & Lunge & 15 & 4 & $1^{\prime}$ & BW \\
\hline & Push up & 10 & 4 & $1^{\prime}$ & BW & Chair dips & 10 & 4 & $1^{\prime}$ & BW \\
\hline & Australian pull up & 15 & 4 & 1'30" & BW & Dumbbell row & 12 & 4 & $1^{\prime}$ & $5+5 \mathrm{~kg}$ \\
\hline & Plank position & $30 "$ & 4 & 1'30" & BW & Sit up (Db) & 20 & 4 & 1'30" & $5 \mathrm{~kg}$ \\
\hline & Hollow position & $30 "$ & 4 & 1'30" & BW & Turkish get up (Db) & 7 & 4 & 1'30" & $5 \mathrm{~kg}$ \\
\hline \multicolumn{11}{|c|}{ INTENSIVE PHASE } \\
\hline \multicolumn{6}{|c|}{ TRAINING 1} & \multicolumn{5}{|c|}{ TRAINING 2} \\
\hline \multirow{8}{*}{ 4-6 weeks } & Exercises & Reps & Set & $\operatorname{Rec}$ & Load & Circuit Training & Time & & Rounc & \\
\hline & Good morning (slow) & 5 & 4 & $2^{\prime}$ & $5 \mathrm{~kg}$ & & & & & \\
\hline & Front squat & 8 & 4 & $2^{\prime}$ & $5+5 \mathrm{~kg}$ & 10 wall ball & & & & \\
\hline & Military press & 12 & 4 & $2^{\prime}$ & $5+5 \mathrm{~kg}$ & 12 burpees & minutes & Alvi & As mossit & y rounas \\
\hline & Chest fly reverse & 10 & 4 & $2^{\prime}$ & $5+5 \mathrm{~kg}$ & 25 sit up & & & & \\
\hline & Australian pull up & 20 & 4 & $2^{\prime}$ & $\mathrm{BW}$ & & & & & \\
\hline & Chest fly & 10 & 4 & $2^{\prime}$ & $5+5 \mathrm{~kg}$ & $\begin{array}{c}\text { Specific tennis skills } \\
\text { training }\end{array}$ & & & ne & \\
\hline & Russian twist & 20 & 4 & 1'30" & $5 \mathrm{~kg}$ & $\begin{array}{c}\text { Forehand/backhand } \\
\text { Technique }\end{array}$ & & $10 \mathrm{r}$ & nutes & \\
\hline & & & & IN' & ENSIVE I & ASE & & & & \\
\hline & TRAI & ING 1 & & & & & RAININ & & & \\
\hline & Circuit Training & Time & & Rou & & $\begin{array}{l}\text { Specific tennis skills } \\
\text { training }\end{array}$ & & & ne & \\
\hline & & & & & & $\begin{array}{c}\text { Forehand/backhand } \\
\text { technique }\end{array}$ & & $10 \mathrm{r}$ & nutes & \\
\hline & $10 \mathrm{CMVJ}$ & In the & & & & Short games & & $10 \mathrm{r}$ & nutes & \\
\hline & 20 lunge & possible & & 4 rou & & Tennis tactics & & $10 \mathrm{r}$ & nutes & \\
\hline 7-9 weeks & 25 sit up & time & & & & & & & & \\
\hline & Interval Training & Time & & Rou & & & & & & \\
\hline & Step up & $\begin{array}{l}10 " \text { on } \\
10 " \text { off }\end{array}$ & & $6 \mathrm{rou}$ & & & & & & \\
\hline & Lateral step up & $\begin{array}{l}10 " \text { on } \\
10 " \text { off }\end{array}$ & & $6 \mathrm{rou}$ & & & & & & \\
\hline
\end{tabular}


Table 5 Continued

\begin{tabular}{|c|c|c|c|c|c|c|c|c|c|c|}
\hline \multicolumn{11}{|c|}{ Group B - Intermediate } \\
\hline \multicolumn{11}{|c|}{ PREPARATORY PHASE } \\
\hline \multicolumn{6}{|c|}{ TRAINING 1} & \multicolumn{5}{|c|}{ TRAINING 2} \\
\hline \multirow{6}{*}{$1-3$ weeks } & Exercises & Reps & Set & $\operatorname{Rec}$ & Load & Exercises & Reps & Set & $\operatorname{Rec}$ & Load \\
\hline & Air squat & 15 & 6 & $1^{\prime}$ & BW & Lunge & 15 & 6 & $1^{\prime}$ & BW \\
\hline & Push up & 10 & 6 & $1^{\prime}$ & BW & Chair dips & 10 & 6 & $1^{\prime}$ & BW \\
\hline & Australian pull up & 15 & 5 & $10^{\prime \prime}$ & $\mathrm{BW}$ & Dumbbell row & 12 & 6 & $1^{\prime}$ & $5+5 \mathrm{~kg}$ \\
\hline & Plank position & $45^{\prime \prime}$ & 5 & 1'30" & BW & Sit up (Db) & 20 & 5 & 1'30" & $5 \mathrm{~kg}$ \\
\hline & Hollow position & $45^{\prime \prime}$ & 5 & $1 ' 30^{\prime \prime}$ & BW & Turkish get up (Db) & 7 & 5 & $1 ' 30^{\prime \prime}$ & $5 \mathrm{~kg}$ \\
\hline \multicolumn{11}{|c|}{ INTENSIVE PHASE } \\
\hline \multicolumn{6}{|c|}{ TRAINING 1} & \multicolumn{5}{|c|}{ TRAINING 2} \\
\hline \multirow{8}{*}{ 4-6 weeks } & Exercises & Reps & Set & $\operatorname{Rec}$ & Load & Circuit Training & Time & & Rounc & \\
\hline & Good morning (slow) & 5 & 4 & $2^{\prime}$ & $5 \mathrm{~kg}$ & \multirow{5}{*}{$\begin{array}{l}10 \text { wall ball } \\
12 \text { burpees } \\
25 \text { sit up }\end{array}$} & \multirow{5}{*}{$\begin{array}{c}12 \\
\text { minutes }\end{array}$} & \multirow{5}{*}{\multicolumn{3}{|c|}{$\begin{array}{l}\text { AMRAP (As many rounds } \\
\text { as possible) }\end{array}$}} \\
\hline & Front squat & 8 & 6 & $2^{\prime}$ & $5+5 \mathrm{~kg}$ & & & & & \\
\hline & Military press & 12 & 6 & $2^{\prime}$ & $5+5 \mathrm{~kg}$ & & & & & \\
\hline & Chest fly reverse & 10 & 5 & $2^{\prime}$ & $5+5 \mathrm{~kg}$ & & & & & \\
\hline & Australian pull up & 20 & 6 & $2^{\prime}$ & BW & & & & & \\
\hline & Chest fly & 10 & 5 & $2^{\prime}$ & $5+5 \mathrm{~kg}$ & $\begin{array}{l}\text { Specific tennis skills } \\
\text { training }\end{array}$ & \multicolumn{4}{|c|}{ Time } \\
\hline & Russian twist & 20 & 5 & 1'30" & $5 \mathrm{~kg}$ & $\begin{array}{c}\text { Forehand/backhand } \\
\text { technique }\end{array}$ & \multicolumn{4}{|c|}{15 minutes } \\
\hline \multicolumn{11}{|c|}{ INTENSIVE PHASE } \\
\hline \multicolumn{6}{|c|}{ TRAINING 1} & \multicolumn{5}{|c|}{ TRAINING 2} \\
\hline \multirow{8}{*}{ 7-9 weeks } & Circuit Training & Time & & Rour & & $\begin{array}{l}\text { Specific tennis skills } \\
\text { training }\end{array}$ & & & ne & \\
\hline & \multirow{4}{*}{$\begin{array}{l}\text { 1' jump rope } \\
10 \text { CMVJ } \\
20 \text { lunge } \\
7 \text { push up } \\
25 \text { sit up }\end{array}$} & \multirow{4}{*}{$\begin{array}{l}\text { In the } \\
\text { shortest } \\
\text { possible } \\
\text { time }\end{array}$} & \multirow{4}{*}{\multicolumn{3}{|c|}{6 rounds }} & $\begin{array}{c}\text { Forehand/backhand } \\
\text { Technique }\end{array}$ & \multicolumn{4}{|c|}{15 minutes } \\
\hline & & & & & & Short Games & \multicolumn{4}{|c|}{15 minutes } \\
\hline & & & & & & Tennis Tactics & \multicolumn{4}{|c|}{15 minutes } \\
\hline & & & & & & & & & & \\
\hline & Interval Training & Time & \multicolumn{3}{|c|}{ Round } & & & & & \\
\hline & Step up & $\begin{array}{l}10 " \text { on } \\
10 " \text { off }\end{array}$ & & 8 rou & & & & & & \\
\hline & Lateral step up & $\begin{array}{l}10 " \text { on } \\
10 " \text { off }\end{array}$ & & 8 rou & & & & & & \\
\hline & & & & Group & and $D$ - & vanced & & & & \\
\hline & & & & PREP & RATOR & HASE & & & & \\
\hline & TRAI & ING 1 & & & & & RAININ & & & \\
\hline & Exercises & Reps & Set & $\operatorname{Rec}$ & Load & Exercises & Reps & Set & $\operatorname{Rec}$ & Load \\
\hline & Air squat & 15 & 8 & $1^{\prime}$ & BW & Lunge & 15 & 8 & $1^{\prime}$ & BW \\
\hline 10 & Push up & 10 & 8 & $1^{\prime}$ & BW & Chair dips & 10 & 8 & $1^{\prime}$ & BW \\
\hline & Australian pull up & 15 & 6 & 1'30" & BW & Dumbbell row & 12 & 8 & $1^{\prime}$ & $5+5 \mathrm{~kg}$ \\
\hline & Plank position & $60^{\prime \prime}$ & 6 & 1'30" & BW & Sit up (Db) & 20 & 6 & 1'30" & $5 \mathrm{~kg}$ \\
\hline & Hollow position & $60^{\prime \prime}$ & 6 & 1'30" & BW & Turkish get up (Db) & 7 & 6 & 1'30" & $5 \mathrm{~kg}$ \\
\hline
\end{tabular}


Table 5 Continued

\begin{tabular}{|c|c|c|c|c|c|c|c|c|}
\hline \multicolumn{9}{|c|}{ INTENSIVE PHASE } \\
\hline \multicolumn{6}{|c|}{ TRAINING 1} & \multicolumn{3}{|c|}{ TRAINING 2} \\
\hline \multirow{8}{*}{ 4-6 weeks } & Exercises & Reps & Set & $\operatorname{Rec}$ & Load & Circuit Training & Time & Round \\
\hline & Good morning (slow) & 5 & 6 & $2^{\prime}$ & $5 \mathrm{~kg}$ & \multirow{5}{*}{$\begin{array}{l}10 \text { wall ball } \\
12 \text { burpees } \\
25 \text { sit up }\end{array}$} & \multirow{5}{*}{$\begin{array}{c}12 \\
\text { minutes }\end{array}$} & \multirow{5}{*}{$\begin{array}{c}\text { AMRAP (As many rounds } \\
\text { as possible) }\end{array}$} \\
\hline & Front squat & 8 & 8 & $2^{\prime}$ & $5+5 \mathrm{~kg}$ & & & \\
\hline & Military press & 12 & 8 & $2^{\prime}$ & $5+5 \mathrm{~kg}$ & & & \\
\hline & Chest fly reverse & 10 & 5 & $2^{\prime}$ & $5+5 \mathrm{~kg}$ & & & \\
\hline & Australian pull up & 20 & 8 & $2^{\prime}$ & BW & & & \\
\hline & Chest fly & 10 & 5 & $2^{\prime}$ & $5+5 \mathrm{~kg}$ & $\begin{array}{c}\text { Specific tennis skills } \\
\text { training }\end{array}$ & & Time \\
\hline & Russian twist & 20 & 6 & 1'30" & $5 \mathrm{~kg}$ & $\begin{array}{c}\text { Forehand/backhand } \\
\text { Technique }\end{array}$ & & 20 minutes \\
\hline \multicolumn{9}{|c|}{ INTENSIVE PHASE } \\
\hline \multicolumn{6}{|c|}{ TRAINING 1} & \multicolumn{3}{|c|}{ TRAINING 2} \\
\hline \multirow{8}{*}{ 7-9 weeks } & Circuit Training & Time & & Rot & & $\begin{array}{l}\text { Specific tennis skills } \\
\text { training }\end{array}$ & & Time \\
\hline & \multirow{4}{*}{$\begin{array}{l}\text { 1' jump rope } \\
10 \text { CMVJ } \\
20 \text { lunge } \\
7 \text { push up } \\
25 \text { sit up }\end{array}$} & \multirow{4}{*}{$\begin{array}{l}\text { In the } \\
\text { shortest } \\
\text { possible } \\
\text { time }\end{array}$} & \multirow{4}{*}{\multicolumn{3}{|c|}{8 rounds }} & $\begin{array}{c}\text { Forehand/backhand } \\
\text { technique }\end{array}$ & & 20 minutes \\
\hline & & & & & & Short games & & 20 minutes \\
\hline & & & & & & Tennis tactics & & 20 minutes \\
\hline & & & & & & & & \\
\hline & Interval Training & Time & \multicolumn{3}{|c|}{ Round } & & & \\
\hline & Step up & $\begin{array}{l}10^{\prime \prime} \text { on } \\
10 " \text { off }\end{array}$ & \multicolumn{3}{|c|}{10 rounds } & & & \\
\hline & Lateral step up & $\begin{array}{l}10^{\prime \prime} \text { on } \\
10^{\prime \prime} \text { off }\end{array}$ & \multicolumn{3}{|c|}{10 rounds } & & & \\
\hline
\end{tabular}

The structuring of the activities basically followed two steps of investigations and one of re-elaboration:

A first phase of investigation in which proceeded to the detection (pre the lockdown due to COVID-19) of some parameters related to Heart Rate, using maximum shooting tests on the 300 meters, reproduced in the same way by all the participants in the project (i.e. environmental conditions, physical fatigue, rest). Information about the environments, tools and spaces already available within each their homes were asked.

In this second phase, following the investigations carried out on the family environments and on the physiological characteristics of each participating subject, coaches were able to start elaborating the different adapted activities and creating diversified groups.

A third phase of investigation in which it was administered a questionnaire (by phoning and video call) to each athlete who participated in. The questionnaire was structured as a Visual Analog Scale (VAS), able to evaluate the subjective perception. In fact, it has been reported that an ordinal scale based on subjective assessments, also used in the clinical and research fields, is a very commonly used tool for monitoring the subject's evolution in physical therapy. Moreover, it is applicable in many circumstances and it is a good method of assessing an athlete's response training [37]. The survey was tested with the following questions:

1. How is your general satisfaction respect to conceived/designed training protocol?

2. Did the proposed exercises have been clearly explained by the instructor?

3. Did the proposed exercises prove to be adequate for your group and play level, in view of an imminent return to the field?

4. Were the spaces that you have available sufficient and adequate for carrying out all the proposed activities?

5. Was the choice of training methodology (circuit work) effective and stimulating for you?

6. In general, do you feel adequately prepared for a possible return to the playing field in the coming weeks?

7. Do you feel adequately prepared for a possible return to the playing field in the coming weeks, from a physical point of view?

8. Do you feel adequately prepared for a possible return to the playing field in the coming weeks, from a technical point of view?

9. Do you feel adequately prepared for a possible return to the playing field in the coming week, from a tactical point of views? 


\section{How do you rate this distance training experience?}

The questionnaire was administered to athletes at $\mathrm{T} 1$, $\mathrm{T} 2, \mathrm{~T} 3$ and $\mathrm{T} 4$.

The athletes with age of majority, involved in this study gave written informed consent, obtained through use of e-mail, in respecting the ethical principles of the Declaration of Helsinki, when minors athletes the signed written parental consents were obtained.

\section{Statistical Analysis}

All data were collected using an online questionnaire and video monitoring at different time points, where due tabulated in a excel spreadsheet (Microsoft, Washington, WA, USA) and analyzed using GraphPad Prism 9. 0. 2 (GraphPad So ware Inc., San Diego, CA, USA).

Considering the low number of athletes, we performed also the descriptive statistics. Percentage changes, defined as [(post-pre)/pre)*100], were used for the analysis to assess home-training effects.

Anthropometric and physiological parameters, were analyzed by non-parametric test, Friedman and post hoc Dunn's multiple comparison test was used to verify significant differences at the different time-points.
Significant level was set at $\mathrm{p}<0.05$. Data are reported as [mean $\pm \mathrm{SD}]$.

\section{Results}

During the two months, the young athletes added some $\mathrm{mm}$ to their height. Statistic tests show the significant difference in groups $\mathrm{A}$ and $\mathrm{C} \quad(\mathrm{p}<0.01$ and $\mathrm{p}<0.02$ respectively), but post hoc does not show the significant difference among time-point (Figure 1a). However, between $\mathrm{T} 1$ and $\mathrm{T} 4+8 \mathrm{~mm}$ of growth in both groups were reported.

Also, a significant increase was observed in change of weight in group $\mathrm{C}$ at $\mathrm{T} 4$ respect $\mathrm{T} 1$ ( $71.8 \pm 6.7$ vs $69.8 \pm 6.8$ $\mathrm{kg}$, respectively, Figure 1b), while, not significant differences were observed in BMI (Figure 1c) among groups.

HRmax significantly decreased at $\mathrm{T} 4$ respect $\mathrm{T} 1$ in all groups (Friedman $\mathrm{p}<0.05$ ), but only post-hoc test was significant in D group (188.6 \pm 5.9 vs $190.8 \pm 5.5)$, Figure 1d).

No significant differences in $\mathrm{SaO}_{2}$ were recorded, where the mean value was $99.2 \pm 0.5 \%$.

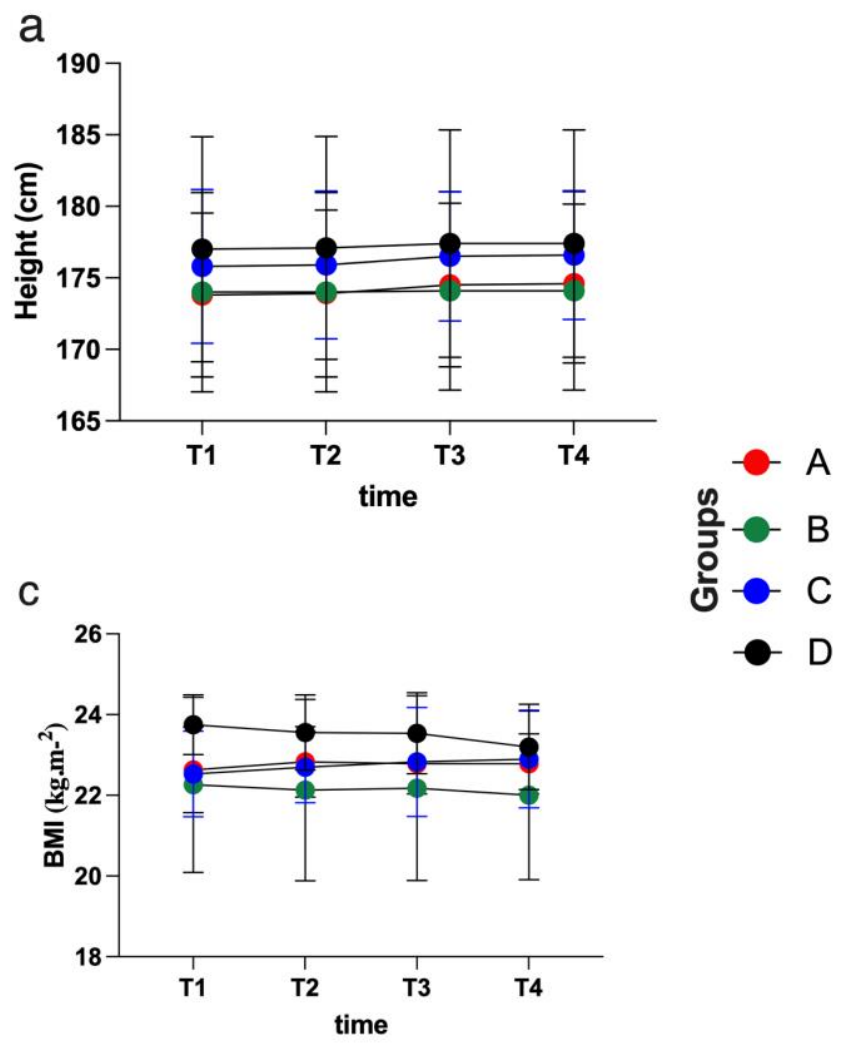

b
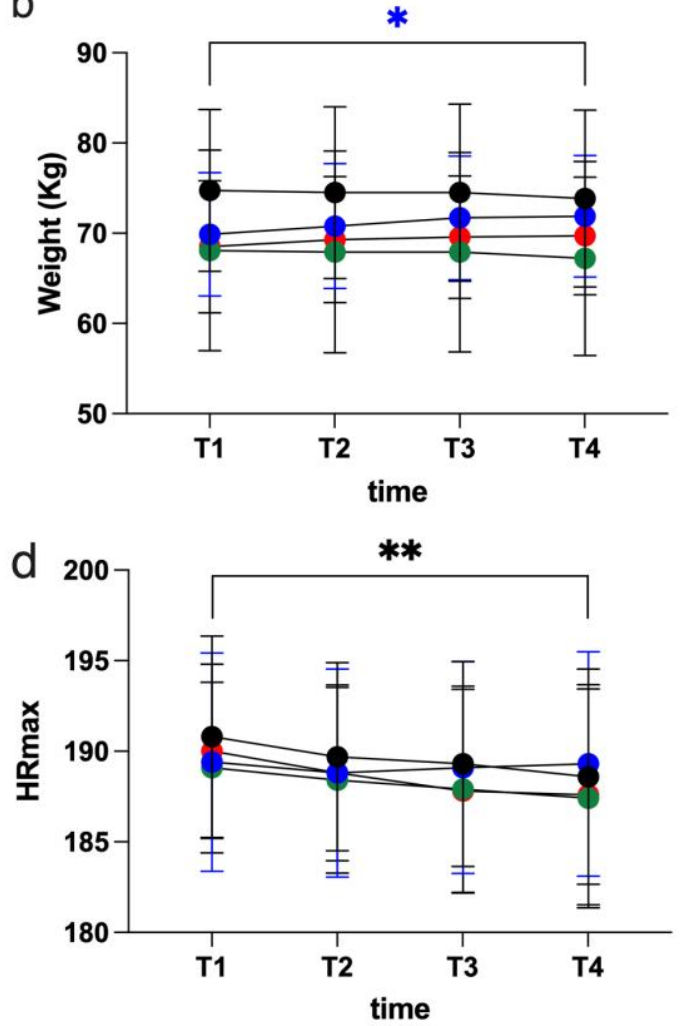

Figure 1. Trend in four groups of: a) height, b) weight, c) BMI, and d) HRmax during the lockdown. *Significant difference within the group (p<0.01); In the figure, the legend with the colors of the four groups of athletes and their respective statistical significances in the same color as the groups 


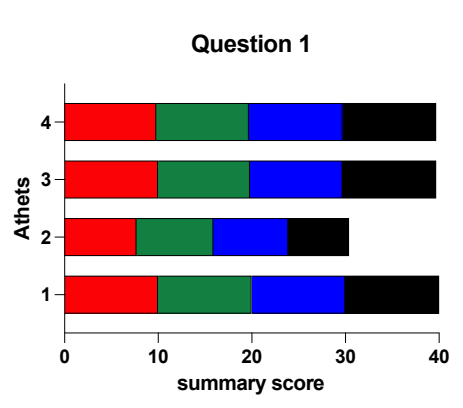

Question 4

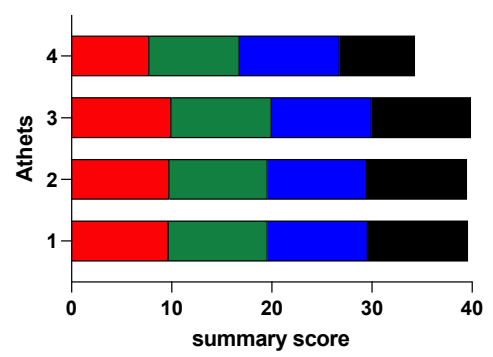

Question 7

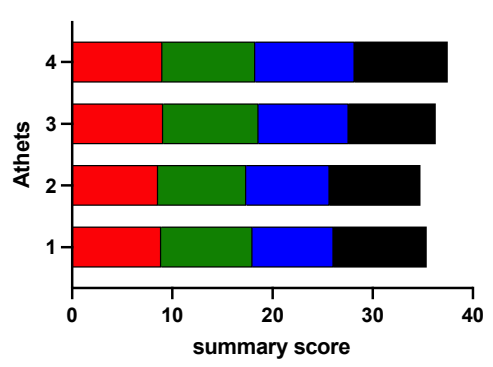

Question 10

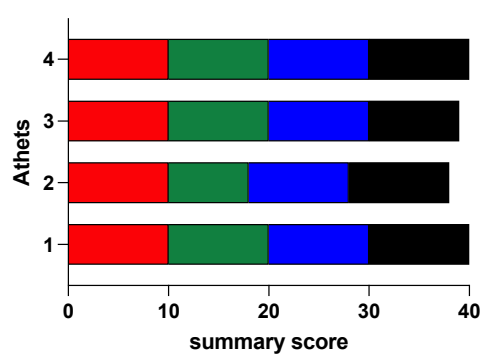

Question 2

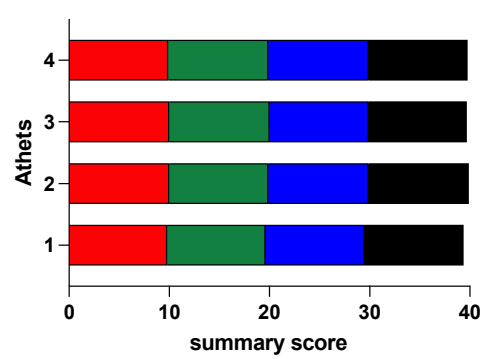

Question 5

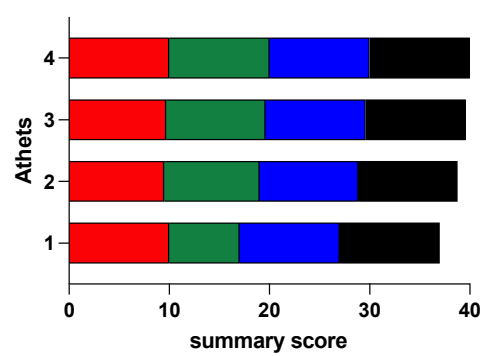

Question 8

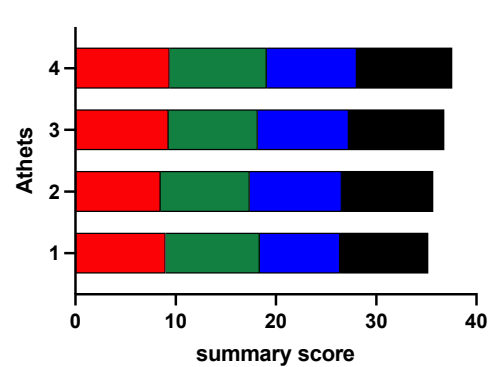

Question 3

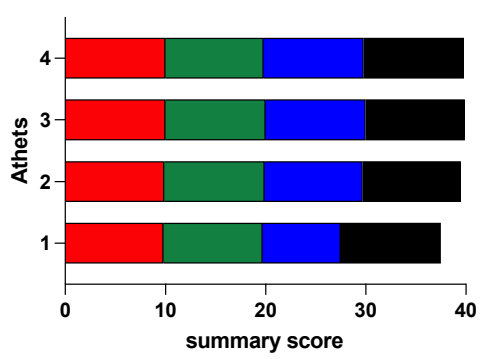

Question 6

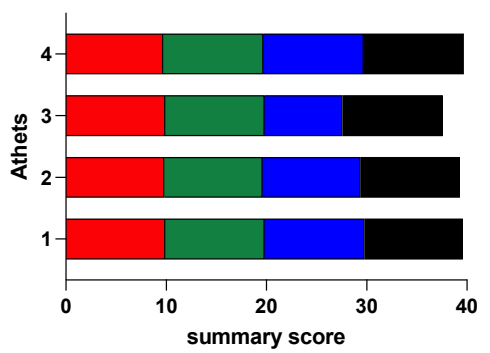

Question 9

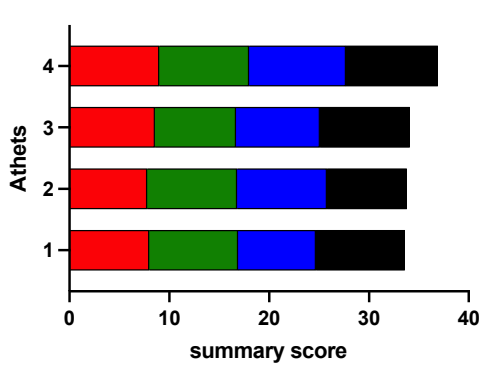

Figure 2. Plot grouped summary data of the feedback received from athletes regard the question on satisfaction of remote-home-training.

At the end of the remote-home-training, the feedback received from athletes, by VAS was positive, reaching all the objectives.

In details (Figure 2), athletes reported:

1. General satisfaction respect to training protocol: group A 93.5\%, B 94.7\%, C 94.7\%, D 91.2\%.

2. Personal satisfaction for proposed exercises and clearness of explanation by the instructor: group A $99 \%$, B $99 \%$, C $99.5 \%$, D $99.5 \%$.
3. Consensus for the proposed exercises respect to the group and the play level, in view of an imminent return to the field: group A 99\%, B 99\%, C 94\%, D $99 \%$.

4. Consensus for the available and adequate spaces at home for carrying out all the proposed activities: group A 93\%, B 96,7\%, C 99,7\%, D 93,5\%.

5. Consensus respect the training methodology (circuit work): group A 98\%, B 91\%, C 99.7\%, D 99.7\%. 
6. General evaluation on preparation respect to a possible return to the playing field in the coming weeks: group A 98.2\%, B 99\%, C 94\%, D 99.2\%.

7. Evaluation on physical preparation respect to a possible return to the playing field in the coming weeks: group A 89\%, B 91.7\%, C 88.2\%, D $91 \%$.

8. Evaluation on technical preparation respect to a possible return to the playing field in the coming weeks: group A 90\%, B 92.2\%, C 88.2\%, D 92.2\%.

9. Evaluation on tactical preparation respect to a possible return to the playing field in the coming weeks: group A 83.5\%, B 87,5\%, C 87.2\%, D 87.7\%.

10. General evaluation on the home-training experience: group A 100\%, B 95\%, C 100\%, D 97.5\%.

\section{Discussion}

It is widely known that physical activity and training have beneficial effects on physical, mental, and social health $[18,19,23]$; in particular, tennis and other rackets sports can be played by anyone at any level without distinction for age or sex [38]. The unexpected lockdown, due to the COVID-19 emergence, has destabilized all the population and especially the athletes, leading to decrease physical activity and the consequent well-being [39,40].

The current study reports the home-training perceptions of satisfaction of athletes during a "first lockdown in Italy in 2020".

The lockdown could have some negative effects on mental, emotional health, stress, and training motivation as reported [38,39,40,41,42] This because athletes experience an uncertainty about their return to competition and their performance level [43]. COVID-19 not only completely changed our lives but also took us by surprise. Having been taken by surprise, from one day to the next, we had to reinvent ourselves in order to survive, trying to continue the work that had been abruptly interrupted and trying to lighten the days of the young athletes. For this reason, with the means available and technology (i.e., smartphone, tablet,), we thought about this form of home-training.

It was not thought to be a success, as it turned out because the athletes may prefer the physical presence of their coach, as training at a medium-high level on your own isn't psychologically easy. Meeting the hands-on needs of instruction was be a challenge.

Not having any more available fields for carrying out training, the coach decided to elaborate and design training protocols as close as possible and corresponding to the technical-tactical-metabolic requirements of this sport, in order to make the experience as similar as possible to routine training, but obviously without using the ordinary surface and avoiding all direct interaction with other companions. We are aware that remote coaching had and has drawbacks and some limitations, but alas, it was and is the unique opportunity for both coaches and athletes during the COVID-19 pandemic, in particular during the first Italian lockdown in 2020.

The main question to solve was: how could it be possible to think of training perfectly respondent to the characteristics that a tennis lesson must have and, at the same time, be adapted to each subject involved according to his/her age, level of play, equipment and spaces available at home?

The answer certainly required an initial analysis of the characteristics of the athletes who participated in the project and, secondly, investigations into the familiar environments and the tools available. Therefore, this project firstly "studied" the participants by monitoring different metabolic and physical parameters, including BMI, Heart Rate max, Heart Rate at rest, reserve Heart Rate, and then subjected them to some questionnaires in order to have a sort of "inventory" ready of the material with which start work on.

The study of the technical-metabolic characteristics of the subjects participating in the project was certainly accompanied by a preliminary investigation on the actual availability of the same and precisely on the space-time availability (rooms in the house, tools, and free days), for which also the participants had to show their approval and their commitment to get involved. The experience to practice distance training for an indeterminate time was accepted by the subjects involved as a chance to continue training despite the forced lockdown.

Boys and girls have decided to rely on this project totally, without time limitations and with the pure desire to savor relative normality, which represents an outlet, of emotion and fun, that is sport.

Recent research in the field of sports medicine has also raised concerns about the impact of coronavirus disease on athletes' health. As reported in the literature [44,45] long-term detraining could bring a loss of endurance capacity and a loss of muscle strength and mass. These factors will significantly increase the risk of injury amongst athletes.

Lockdown period has presented absolutely unprecedented situation for all people and certainly a dramatic shift in teaching, and in the learning [46]. This was not the case only for the education (primary/higher) assessment practices, but also for all that is the recreational and sporting sphere.

The athletes involved in this study have demonstrated, by means of the questionnaire (VAS), the beneficial effects of home-training during the lockdown both on physical (e.g. HR measures might reflect (aerobic-based) adaptation and fatigue status, thence are able to represent the athlete's training status [47]) and mental well-being (Figure 3), even if, as we have been able to see from the answers, the greatest doubts remain on the technical and tactical preparation. However, our preliminary findings provide further insight compared to "outdoor" training methods. 


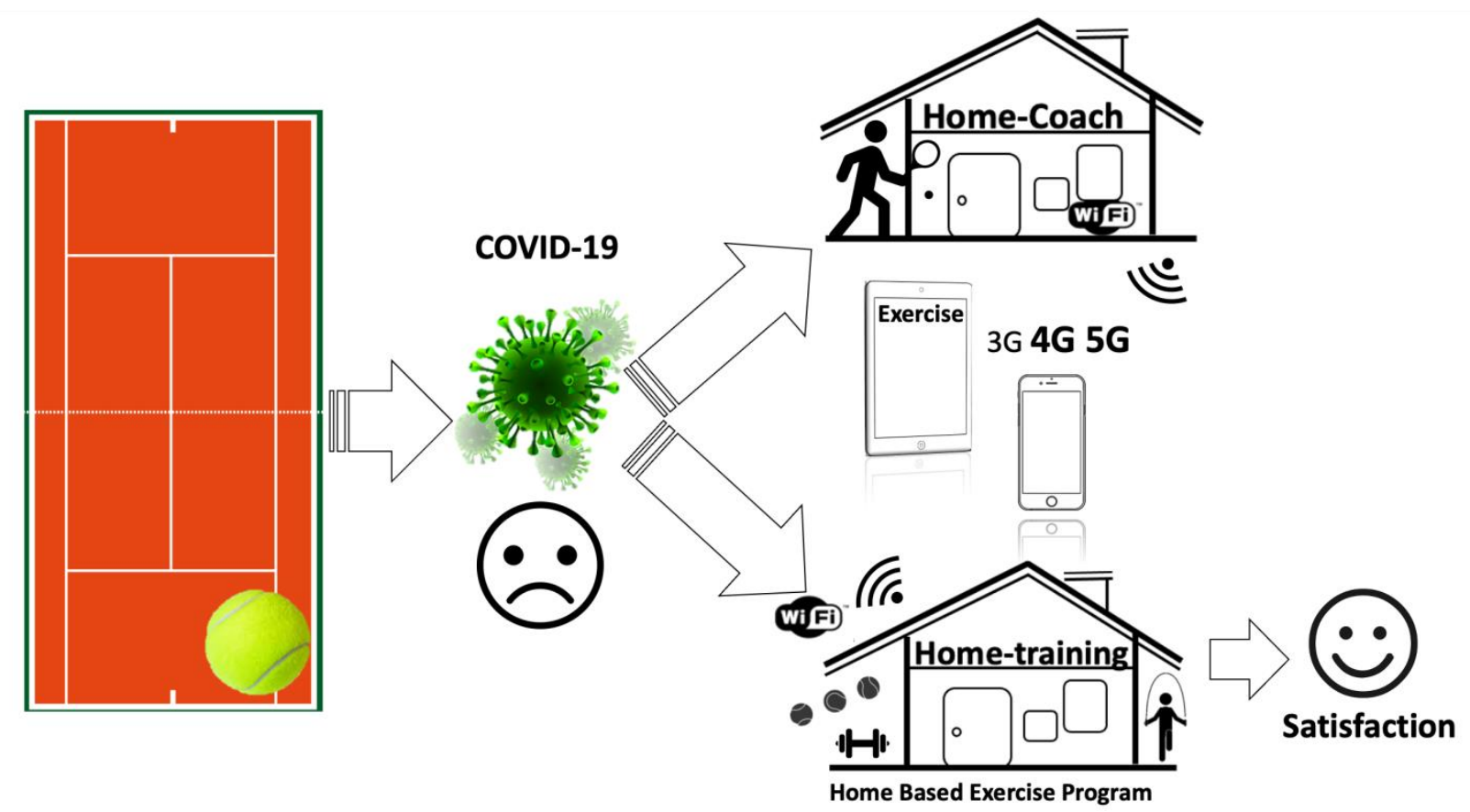

Figure 3. Scheme of tennis home-training and the general satisfaction in young athletes during first Italy lockdown COVID-19

\section{Limitations}

The authors are aware that the current study suffers from certain limitations as the sample size and the lack of any control group. However, we would like to emphasize that the coach-team consisted of 16 young athletes, and that everyone participated in the study. Athletes were divided into four groups according to the level of play and the pre-existing technical-tactical preparation, all have been included and analyzed in this pilot study. Given the scarcity of research including young athletes [48], and in particular tennis players, the sample size was been determined by accessibility. Because of the influence posed by this pandemic, it is important to use caution without generalizing when interpreting the data. As such, future researches in case of other blocking periods must extend the current findings.

Finally, we conclude that no measures were done when the athletes returned to the playing field after lockdown and that future studies may use a mixed approaches to confirm the current study's which was suggested in previous studies [19].

\section{Conclusions}

In conclusion, we can say that the remote home-training, offered athletes one option to maintain both "normal" training practices, in order to avoid a risk of detraining in athletes in view of a return to the tennis court.

Anyway, further studies, with the use of implemented platforms could be more appropriate and yield novel training programs in sports. To date, COVID-19 pandemic represents a global crisis that has forced athletes young/elite/not and coaches into an unknown situation.

\section{Author Contributions}

Conceptualization, A.S., S.M.S.; methodology, A.S., R.D.D., S.M.S.; formal analysis, A.S., G.DA., R.D.D., S.M.S.; investigation; A.S.; data curation, A.S., M.M., A.V., S.M.S.; writing - original draft preparation, A.S., M.M., R.D.D., S.M.S.; writing - review and editing, A.S., M.M., A.V., G.DA., S.M.S.; visualization, S.M.S; supervision, S.M.S project administration: A.S., S.M.S. All authors have read and agreed to the published version of the manuscript.

\section{Funding}

This research received no external funding.

\section{Institutional Review Board Statement}

The study was conducted according to the guidelines of the Declaration of Helsinki.

\section{Informed Consent Statement}

Informed consent was obtained from all coaches involved in the study. Written informed consent has been obtained from the coaches to publish all data is available 
within the manuscript.

\section{Data Availability Statement}

All data is available within the manuscript.

\section{Acknowledgments}

We would like to express our sincere gratitude and thanks to all athletes.

\section{Conflicts of Interest}

The authors declare no conflict of interest.

\section{REFERENCES}

[1] World Health Organization, "Novel Coronavirus (2019-nCoV) technical guidance", 2020. https://covid19.who.int/.

[2] Garcia-Garcia B., James D., Koller M., Lindholm J., Mavromati D., Parrish R., R. Rodenberg, "The impact of Covid-19 on sports: a mid-way assessment," The International Sports Law Journal, vol. 20, pp. 115-119, 2020. DOI: $10.1007 / \mathrm{s} 40318-020-00174-8$.

[3] Parnell D., Widdop P., Bond A., R. Wilson, "COVID-19, network and sport," Managing Sport and Leisure. 2020. DOI: $1080 / 23750472.2020 .1750100$.

[4] V. Ratten, "Coronavirus disease (COVID-19) and sport entrepreneurship," International Journal of Entrepreneurial Behavior \& Research, vol. 26, no. 6, pp. 1356-2554, 2020. DOI: 10.1108/IJEBR-06-2 020-0387.2020.

[5] Zhang C., Ye M., Fu Y., Yang M., Luo F., Yuan J., Q. Tao, "The psychological impact of the COVID-19 pandemic on teenagers in China," Journal of Adolescent Health, vol. 67, no. 6 , pp. 747-755, 2020. DOI: $10.1016 /$ j.jadohealth. 2020 . 08.026

[6] Costituzione italiana. Available online at: https://www.governo.it/it/costituzione-italiana/principi-fon damentali/2839.

[7] Hawkley L. C., J. P. Capitanio, "Perceived social isolation, evolutionary fitness and health outcomes: a lifespan approach," Philosophical Transactions of the Royal Society B: Biological Sciences, vol. 370, pp. 1669, 2015. DOI: 10.1098/rstb.2014.0114.

[8] Rodríguez-Rey R., Garrido-Hernansaiz H., S. Collado, "Psychological impact and associated factors during the initial stage of the coronavirus (COVID-19) pandemic among the general population in Spain," Frontiers in psychology, vol. 11, pp. 1540, 2020. DOI: 10.3389/fpsyg.2020.01540.

[9] Franco I., Bianco A., Bonfiglio C., Sorino P., Mirizzi A.,
Campanella A., A. R. Osella, "Decreased levels of physical activity: results from a cross-sectional study in southern Italy during the COVID-19 lockdown," The Journal of sports medicine and physical fitness, vol. 61, no. 2, pp. 294-300, 2021. DOI: 10.23736/S0022-4707.20.11536-6.

[10] Ruberti O. M., Telles G. D., B. Rodrigues, "Stress and physical inactivity: two explosive ingredients for the heart in COVID-19 pandemic times", Current Cardiology Reviews, 2021. DOI: 10.2174/1573403X17666210126103 204.

[11] Chtourou H., Trabelsi K., H'mida C., Boukhris O., Glenn J. M., Brach M., N. L. Bragazzi, "Staying physically active during the quarantine and self-isolation period for controlling and mitigating the COVID-19 pandemic: a systematic overview of the literature," Frontiers in psychology, vol. 11 pp. 1708, 2020. DOI: 10.3389/fpsyg.2020.01708.

[12] Ingram J., Maciejewski G., C.J. Hand, Changes in diet, sleep, and physical activity are associated with differences in negative mood during COVID-19 lockdown. Frontiers in psychology, vol. 11, pp. 2328, 2020. DOI: 10.3389/fpsyg.2020.588604.

[13] López-Bueno R., Calatayud J., Casaña J., Casajús, J. A., Smith L., Tully M. A., G.F. López-Sánchez, "COVID-19 confinement and health risk behaviors in Spain," Frontiers in Psychology, vol. 11, pp. 1426, 2020. DOI: 10.3389/fpsyg.2020.01426.

[14] Hytner M., B. Butler, "Quantas Ends 30-Year Wallabies Sponsorship Deal in Huge Blow to Rugby Australia,” 2020 Available online at: https://www.theguardian.com/sport/20 20/sep/23/qantas-ends-30-year-sponsorship-deal-in-huge-b low-for-rugby-australia.

[15] McCurry J., S. Ingle, "Tokyo Olympics postponed to 2021 due to coronavirus pandemic; 24.03. 2020". Available online at: https://www.theguardian.com/sport/2020/mar/24 /tokyo-olympics-to-be-postponed-to-2021-due-to-coronavi rus-pandemic.

[16] O’Connor C. (2020). "Devastated Rower Say Olympic Postponement Hard to Accept But The Correct Decision". Available online at: https://www.irishexaminer.com/sport/ arid-30989823.html.

[17] Bok D., Chamari K., C. Foster, "The pitch invader-COVID-19 canceled the game: what can science do for us, and what can the pandemic do for science?," International journal of sports physiology and performance, vol. 15 , no. 7 , pp. 917-919, 2020. DOI: 10.1123/ijspp.2020-0467.

[18] Gallè F., Sabella E. A., Ferracuti S., De Giglio O., Caggiano G., Protano C., C. Napoli, "Sedentary behaviors and physical activity of Italian undergraduate students during lockdown at the time of CoViD- 19 pandemic," International journal of environmental research and public health, vol. 17, no. 17, pp. 6171, 2020. DOI: 10.3390/ijerph17176171.

[19] Rasheed A., Abduljawad R., Mabrouk S., Jdaitawi M., M. Abdulmonem, "Physical Fitness Training Program Using Electronic Simulation Games to Foster Psychological Health among University Students during COVID-19 Pandemic," International Journal of Human Movement and Sports Sciences, vol. 9, no. 3, pp. 421-427, 2021. DOI: 10.13189/saj.2021.090305. 
[20] MacIntyre T., Brick N., Butler C., Doherty A., Lane A. M., Morris R., M. Rogan, "Beyond the COVID-19 Pandemic: Tips for Players and Athletes COVID-RECOVER," 2020. Available online at: https://www.psychologicalsociety.ie/s ource/Beyond\%20the\%20COVID-19\%20Pandemic\%20Ti ps $\% 20$ for $\% 20$ Players $\% 20$ and $\% 20$ Athletes $\% 20$ COVID-RE COVER.pdf.

[21] Schinke R., Papaioannou A., Maher C., Parham W. D., Larsen C. H., Gordin R., S. Cotterill, "Sport psychology services to professional athletes: working through COVID-19," International Journal of Sport and Exercise Psychology, vol. 18, no. 4, pp. 409-413, 2020. DOI: 10.1080/1612197X.2020.1766182.

[22] Washif J. A., Mohd Kassim S. F. A., Lew P. C. F., Chong C. S. M., C. James, "Athlete's Perceptions of a "Quarantine" Training Camp During the COVID-19 Lockdown," Frontiers in Sports and Active Living, vol. 2, pp. 220, 2021. DOI: $10.3389 /$ fspor.2020.622858.

[23] Malm C., Jakobsson J., A. Isaksson, "Physical activity and sports - real health benefits: a review with insight into the public health of Sweden," Sports, vol. 7, no. 5, pp. 127, 2019. https://doi.org/10.3390/sports7050127.

[24] Janssen I., Roberts K. C., W. Thompson, "Is adherence to the Canadian 24-Hour Movement Behaviour Guidelines for Children and Youth associated with improved indicators of physical, mental, and social health?," Applied Physiology, Nutrition, and Metabolism, vol. 42, no. 7, pp. 725-731, 2017. DOI: 10.1139/apnm-2016-0681.

[25] Garber C. E., Blissmer B., Deschenes M. R., Franklin B. A., Lamonte M. J., Lee I. M., D. P. Swain, "Quantity and quality of exercise for developing and maintaining cardiorespiratory, musculoskeletal, and neuromotor fitness in apparently healthy adults: guidance for prescribing exercise," Medicine \& Science in Sports \& Exercise, vol. 43, no. 7 , pp. 1334-1359, 2011. DOI: 10.1249/MSS.0b013e31 $8213 \mathrm{fefb}$.

[26] De Almeida S. I. L., Gomes da Silva M., A. S. P. D. D. Marques, "Home-based physical activity programs for people with dementia: Systematic review and meta-analysis," The Gerontologist, vol. 60, no. 8, pp. e600-e608, 2020. DOI:10.1093/geront/gnz176.

[27] Loh K. P., Kleckner I. R., Lin P. J., Mohile S. G., Canin B. E., Flannery M. A., K. M. Mustian, Effects of a home based exercise program on anxiety and mood disturbances in older adults with cancer receiving chemotherapy. Journal of the American Geriatrics Society, vol. 67, no. 5, pp. 1005-1011, 2019. DOI:10.1111/jgs.15951.

[28] Chien C. L., Lee C. M., Wu Y. W., Y. T. Wu, "Home-based exercise improves the quality of life and physical function but not the psychological status of people with chronic heart failure: a randomised trial," Journal of physiotherapy, vol. 57, no. 3, pp. 157-163, 2011. DOI: 10.1016/S1836-9553(1 1)70036-4.

[29] Mrakic-Sposta S., Di Santo S.G., Franchini F., Arlati S., Zangiacomi A., Greci L., Moretti S., Jesuthasan N., Marzorati M., Rizzo G., Sacco M., A. Vezzoli, "Effects of Combined Physical and Cognitive Virtual Reality-Based Training on Cognitive Impairment and Oxidative Stress in MCI Patients: A Pilot Study," Front Aging Neurosci. vol. 1, no. 10, pp. 282, 2018. DOI: 10.3389/fnagi.2018.00282.
[30] Vezzoli A., Mrakic-Sposta S., Montorsi M., Porcelli S., Vago P., Cereda F., M. Narici, "Moderate intensity resistive training reduces oxidative stress and improves muscle mass and function in older individuals," Antioxidants, vol. 8, no. 10, pp. 431, 2019. DOI: 10.3390/antiox8100431.

[31] Vizzi L., Padua E., D’Amico A. G., Tancredi V., D'Arcangelo G., Cariati I., M. Montorsi, "Beneficial effects of physical activity on subjects with neurodegenerative disease. Journal of functional morphology and kinesiology, vol. 5, no. 4, pp. 94, 2020. DOI:10.3390/jfmk5040094

[32] [32] Federazione Italiana Tennis (FIT). Available online at: https://www.federtennis.it.

[33] Jackson M. J., Roche D. M., Amirabdollahian F., Koehn S., O. A. Khaiyat, "The Musculoskeletal Health Benefits of Tennis," Sports health, vol. 12, no. 1, pp. 80-87, 2020. DOI: $10.1177 / 1941738119880862$.

[34] Pluim B. M., Groppel J. L., Miley D., Crespo M., M. S. Turner, Health benefits of tennis. British journal of sports medicine, vol. 52 no. 3, pp. 201-202, 2018. DOI: 10.1136/bjsports-2017-098623.

[35] Šlosar L., de Bruin E. D., Fontes E. B., Plevnik M., Pisot R., Simunic B., U. Marusic, "Additional exergames to regular tennis training improves cognitive-motor functions of children but may temporarily affect tennis technique: a single-blind randomized controlled trial," Frontiers in psychology, vol. 12, pp. 783, 2021. DOI: 10.3389/fpsyg.2021.611382.

[36] Shim J., Carlton L. G., Chow J. W., W. S. Chae, "The use of anticipatory visual cues by highly skilled tennis players," Journal of motor behavior, vol. 37, no. 2, pp. 164-175, 2005. DOI: 10.3200/JMBR.37.2.164-175.

[37] Coyne J. O., Haff G. G., Coutts A. J., Newton R. U., S. Nimphius, "The current state of subjective training load monitoring - a practical perspective and call to action," Sports medicine-open, vol. 4, no. 1, pp. 1-10, 2018. DOI: 10.1186/s40798-018-0172-x.

[38] Jayanthi N., S. Esser, "Racket sports," Current sports medicine reports, vol. 12 , no. 5, pp. 329-336, 2013. DOI: 10.1249/JSR.0b013e3182a4bad0.

[39] Maugeri G., G. Musumeci, “Adapted physical activity to ensure the physical and psychological well-being of COVID-19 patients" Journal of Functional Morphology and Kinesiology, vol. 6, no. 1, pp. 13, 2021. DOI: 10.3390/jfmk6010013.

[40] Maugeri G., Castrogiovanni P., Battaglia G., Pippi R., D'Agata V., Palma A., G. Musumeci, "The impact of physical activity on psychological health during Covid-19 pandemic in Italy," Heliyon, vol. 6, no. 6, pp. e0431, 2020. DOI: 10.1016/j.heliyon.2020.e04315.

[41] Ammar A., Brach M., Trabelsi K., Chtourou H., Boukhris O., Masmoudi L., ECLB-COVID19 Consortium, "Effects of COVID-19 home confinement on eating behaviour and physical activity: results of the ECLB-COVID19 international online survey,". Nutrients, vol. 12 , no. 6 , pp. 1583, 2020. DOI: 10.3390/nu12061583.

[42] Pillay L., van Rensburg D. C. C. J., van Rensburg A. J., Ramagole D. A., Holtzhausen L., Dijkstra H. P., T. Cronje, "Nowhere to hide: the significant impact of coronavirus 
disease 2019 (COVID-19) measures on elite and semi-elite South African athletes," Journal of science and medicine in sport, vol. 23, no. 7, pp. 670-679, 2020. DOI: 10.1016/j.jsams.2020.05.016

[43] Andreato L. V., Coimbra D. R., A. Andrade, "Challenges to athletes during the home confinement caused by the COVID-19 pandemic," Strength and Conditioning Journal, vol. 42, no. 3, 2020. DOI: $10.1519 /$ SSC.000000000000056 3.

[44] Baggish A., Drezner J. A., Kim J., Martinez M., J. M. Prutkin, "Resurgence of sport in the wake of COVID-19: cardiac considerations in competitive athletes," British Journal of Sports Medicine, vol. 54, pp. 1130-1131, 2020. DOI: 10.1136/bjsports-2020-102516.

[45] Paoli A., G. Musumeci, "Elite athletes and COVID-19 lockdown: future health concerns for an entire sector," Journal of Functional Morphology and Kinesiology, vol. 5, no. 2 , pp. 30 . DOI: $10.3390 /$ jfmk5020030.

[46] Ritchie L., B. T. Sharpe, "Music Student's Approach to the Forced Use of Remote Performance Assessments," Frontiers in Psychology, vol. 12, pp. 1367, 2021. DOI: 10.3389/fpsyg.2021.641667.

[47] Schneider C., Hanakam F., Wiewelhove T., Döweling A., Kellmann M., Meyer T., Pfeiffer M., A. Ferrauti, "Heart Rate Monitoring in Team Sports-A Conceptual Framework for Contextualizing Heart Rate Measures for Training and Recovery Prescription," Frontiers Physiology, vol. 9, pp. 639, 2018. DOI: 10.3389/fphys.2018.00639.

[48] Dauty M., Menu P., A. Fouasson-Chailloux, "Effects of the COVID-19 confinement period on physical conditions in young elite soccer players," The Journal of sports medicine and physical fitness, vol. Dec. 03, 2020. DOI: 10.23736/s0022-4707.20.11669-4 